\title{
Spikes morphometric characteristics analysis of five species of wheat
}

\author{
Evgeniy Komyshev \\ ICG SB RAS, Kurchatov Genomic \\ Center, Novosibirsk, Russia \\ komyshev@bionet.nsc.ru \\ Yuliya Kruchinina \\ ICG SB RAS, Kurchatov Genomic \\ Center, Novosibirsk, Russia \\ kruchinina2014@bionet.nsc.ru
}

\author{
Mikhail Genaev \\ ICG SB RAS, Kurchatov Genomic \\ Center, Novosibirsk, Russia \\ NSU, Novosibirsk, Russia \\ mag@bionet.nsc.ru \\ Vasiliy Koval \\ ICG SB RAS, Kurchatov Genomic \\ Center, Novosibirsk, Russia \\ kovalvs@icg.sbras.ru
}

\author{
Dmitry Afonnikov \\ ICG SB RAS, Kurchatov Genomic \\ Center, Novosibirsk, Russia \\ NSU, Novosibirsk, Russia \\ ada@bionet.nsc.ru \\ Nikolay Goncharov \\ ICG SB RAS, Novosibirsk, Russia \\ gonch@bionet.nsc.ru
}

\begin{abstract}
A method for recognition and morphometry of a wheat spike based on the analysis of $2 \mathrm{D}$ digital images has been developed. The proposed approach showed high accuracy for determining the qualitative and quantitative characteristics of the spike. An analysis of the obtained characteristics of five species of wheat showed that they are divided into three main clusters characterizing the spike shape.
\end{abstract}

Keywords - image analysis, pattern recognition, morphometry, spikes of wheat

\section{Motivation and aim}

\section{Motivation}

The shape and structure of the spike are one of the most important characteristics of cereals associated with their economically valuable qualities such as productivity, lack of fragility of the spike and ease of threshing. Studying the genes that control these characters will allow to purposefully create new varieties with improved characteristics in terms of yield, ease of threshing and resistance to environmental factors [1]. However, the complexity of research in this area is due to the polyploidy of the wheat genome [2] and the need to obtain phenotypic data from thousands of plants. For example, in the work of Boden et al. [3] to identify loci associated with the structure of a spike (inflorescence), more than 13 thousand spikes were analyzed.

Aim

We recently developed method for morphometry of the wheat spike [4] for analysis of the shape of spikes based on the $2 \mathrm{D}$ image analysis.

We apply our algorithm for analysis of the five species of wheat: Triticum aestivum, Triticum spelta, Triticum compactum, Triticum sphaerococcum, Triticum antiquorum.

\section{Methods}

We propose a wheat spike recognition method based on digital image analysis. Digital images are acquired in two variants: a spike on a table (one projection) or fixed with a clip (four projections). The method identifies spike and awns in the image and estimates their quantitative characteristics (area in image, length, width, circularity, etc.). Models of sections, quadrilaterals, and radial model are proposed for describing spike shape. Parameters of these models are used to predict spike shape type (spelt, normal, or compact) by machine learning.

\section{Results}

This method makes it possible to extract several traits associated with spike shape, its awns, and color characteristics. The proposed approach has shown high performance in identifying the image regions pertaining to the spike and its awns. Several models are proposed for describing the shape of wheat spike.

The mean error in spike density prediction for the images in one projection is $4.61(\sim 18 \%)$ versus $3.33(\sim 13 \%)$ for the parameters obtained using four projections. F1 measure in automated spike classification into three types is 0.78 using logistic regression (one projection) and 0.85 using random forest method (four projections).

The analysis of the obtained characteristics of five wheat of wheat showed that they are divided into three main clusters characterizing the linear dimensions of the spike, area projected by the spike and the shape of the spike contour.

\section{Acknowledgment}

The work supported by the Kurchatov Genomic Center, Institute of Cytology and Genetics, SB RAS, agreement № 075-15-2019-1662 with the Ministry of Education and Science.

\section{References}

[1] Konopatskaia I.D., Vavilova V.Y., Blinov A.G., Goncharov N.P. (2016) Spike morphology genes in wheat species (Triticum L.). Proceedings of the Latvian Academy of Sciences. Section B. Natural, Exact, and Applied Sciences. De Gruyter Open. 70(6): 345-355.

[2] Borrill P., Harrington S. A., Uauy C. (2019) Applying the latest advances in genomics and phenomics for trait discovery in polyploid wheat. The Plant Journal. 97(1): 56-72.

[3] Boden S. A. et al. (2015) Ppd-1 is a key regulator of inflorescence architecture and paired spikelet development in wheat. Nature Plants. 1(2): 14016.

[4] Genaev M.A. et al. (2019) Morphometry of the Wheat Spike by Analyzing 2D Images. Agronomy 9(7): 390. 